\title{
Appetite
}

Appetite 41 (2003) 31-41

www.elsevier.com/locate/appet

Research Report

\section{Disgust sensitivity and meat consumption: a test of an emotivist account of moral vegetarianism}

\author{
Daniel M.T. Fessler*, Alexander P. Arguello, Jeannette M. Mekdara, Ramon Macias \\ Department of Anthropology, Center for Behavior, Evolution, and Culture, UCLA, Los Angeles, CA USA
}

\begin{abstract}
Emotivist perspectives on moral reasoning hold that emotional reactions precede propositional reasoning. Published findings indicate that, compared with health vegetarians, those who avoid meat on moral grounds are more disgusted by meat [Psychol. Sci. 8 (1997) 67]. If, as per emotivist perspectives, such disgust precedes moral rationales for meat avoidance, then the personality trait of disgust sensitivity should generally be inversely related to meat eating. We surveyed 945 adults regarding meat consumption, reasons for meat avoidance, and disgust sensitivity. Contrary to the emotivist prediction, (a) meat consumption was positively correlated with disgust sensitivity, and (b) individuals who reported avoiding meat for moral reasons were not more sensitive to disgust than those who avoided meat for other reasons. We conclude that moral vegetarianism conforms to traditional explanations of moral reasoning, i.e. moral vegetarians' disgust reactions to meat are caused by, rather than causal of, their moral beliefs.

(C) 2003 Elsevier Ltd. All rights reserved.
\end{abstract}

Keywords: Meat consumption; Vegetarianism; Disgust sensitivity; Moral reasoning

\section{Introduction}

Behavioral scientists have long been interested in the relationship between moral beliefs and emotions. Traditional views of moral reasoning (e.g. Kohlberg, 1984; Kurtines \& Gewirtz, 1991; Lapsley, 1996; Turiel, 1983) hold that moral positions are adopted as a result of strictly cognitive processes, with emotions then following in the wake of newly held ideas. In contrast, a growing perspective views complex propositional reasoning as often the consequence, rather than the cause, of emotional responses to the world (cf. Cosmides \& Tooby, 2000; Damasio, 1994; Fessler \& Navarrete, 2003; Greene, Sommerville, Nystrom, Darley, \& Cohen, 2001; Haidt, 2001). The domain of food is one that is typically rich in cultural and personal meanings and is often associated with powerful emotions (Barkow et al., 2001; Bourdieu, 1984; Rozin, 1999; Rozin, Fischler, Imada, Sarubin, \& Wrzesniewski, 1999a; Simoons, 1994). Accordingly, this area provides a potentially productive avenue for investigating the direction of causality in the relationship between beliefs and emotions.

Over the last three decades, moral vegetarianism has become increasingly common in the West. Moral

\footnotetext{
* Corresponding author.

E-mail address: dfessler@anthro.ucla.edu (D.M.T. Fessler).
}

vegetarianism is distinguished from health vegetarianism by virtue of differing justifications of meat avoidance. While health vegetarians avoid meat simply because they believe it is unhealthy, Western moral vegetarians typically link meat consumption to cruelty, environmental degradation, and a variety of political concerns (Adams, 2000; Jabs, Devine, \& Sobal, 1998; Rozin, Markwith, \& Stoess, 1997; cf. Adams, 2000). ${ }^{1}$ Moral vegetarians thus view meat avoidance as a moral imperative and, in contrast to health vegetarians, are upset by others' meat consumption (Rozin et al., 1997). Importantly, the vast majority of moral vegetarians adopt this perspective sometime during adolescence or adulthood (Beardsworth \& Keil, 1992). Moral vegetarianism is thus distinctly different from that large class of beliefs and practices which are acquired through redundant exposure during childhood, a process likely to lead individuals to experience the given ideas as 'transparent' such that it is difficult or impossible to imagine alternatives (cf. Levy, 1973). Western moral vegetarians are acutely aware that they are rejecting the beliefs of the majority culture (Back \& Glasgow, 1981; Beardsworth \& Keil, 1992; Dwyer, Mayer, Dowd, Kandel, \& Mayer, 1974). Because relevant aspects of experience and

\footnotetext{
${ }^{1}$ For information useful in judging the scientific validity of these positions, see A.D.A. (1997), Webster (1994).
} 
behavior are likely to be neither subtle nor difficult to uncover, moral vegetarianism thus constitutes a promising target for the exploration of moral reasoning.

Comparing moral vegetarians and health vegetarians, Rozin et al. (1997) report that moral vegetarians find meat more disgusting (see also Jabs et al., 1998). Disgust is a multifaceted emotion encompassing both a primitive element focusing on revulsion at the prospect of oral incorporation of offensive objects (or similar exposure to contaminants [Curtis \& Biran, 2001], termed core disgust, and symbolically mediated rejections of immoral or polluting objects, behaviors, or persons, termed sociomoral disgust (Haidt, Koller, \& Dias, 1993; Rozin, Haidt, \& McCauley, 1993; Rozin, Haidt, \& McCauley, 1999c). Consistent with traditional approaches to moral reasoning and emotion, Rozin et al. assert that moral vegetarians find meat more disgusting because, having adopted an anti-meat stance on philosophical and ethical grounds, they then (consciously or unconsciously) link meat eating with powerful emotions that provide additional motivational force to their position (see also Rozin \& Singh, 1999). In short, the authors claim that conceptualizing meat eating as immoral creates both an opportunity and an incentive to view meat as disgusting. In contrast to this traditionalist view, an emotivist approach to moral reasoning reverses the causal arrow in this explanation: it is possible that, for many moral vegetarians, meat avoidance is initially motivated by disgust, and the moral stance constitutes a post hoc justification of this emotional response (cf. Haidt et al., 1993; Nisbett \& Wilson, 1977). A variety of findings provide indirect support for this possibility.

Circumstantial evidence indicating that disgust precedes, rather than follows, conversion to moral vegetarianism

Contact with or exposure to animals, death, and body envelope violations are three of the strongest elicitors of disgust (Angyal, 1941; Fallon \& Rozin, 1983; Haidt, McCauley, \& Rozin, 1994; Rozin et al., 1993; Rozin, Haidt, McCauley, \& Dunlop, 1999b). Modern methods of processing, packaging, cooking, and presenting meat remove reminders of the whole animal and eliminate, disguise, or mitigate cues that meat is in fact muscle from the interior of a once-living creature (cf. Beardsworth \& Keil, 1992; Fiddes, 1991, 87-96). Moral vegetarians frequently report a history of highly charged encounters with such normally muted meat-related cues prior to 'turning vegetarian', and there are hints that the road to moral vegetarianism may often begin with a disgust response to specific features of meat (cf. Beardsworth \& Keil, 1992; Jabs et al., 1998; Janda \& Trocchia, 2001; Kubberod, Ueland, Tronstad, \& Risvik, 2002; Santos \& Booth, 1996; Ritson, cited in Simoons, 1994, 11). For example, despite the fact that the logic of moral vegetarianism applies equally to the consumption of any sort of flesh, in Western populations meat avoidance often begins with red meat (typically beef), then progresses to other meats (Kenyon \& Barker, 1998; Santos \& Booth, 1996). ${ }^{2}$ Blood is a powerful stimulus, and the avoidance of red meat frequently stems from revulsion at the presence of blood (Kenyon \& Barker, 1998; Santos \& Booth, 1996) indeed, even those who eat red meat may be disgusted by bloody meat (Beardsworth \& Keil, 1992; Chagnon, 1997, 101, 102; Fiddes 1991, 89, 90; Kenyon \& Barker, 1998; Kubberod et al., 2002; Twigg, 1979; Santos \& Booth, 1996). Pork, poultry, and fish only appear white once the blood has been drained from the tissue, a transformation that artificially alters the meat's evocative power. Although modern meat marketing reduces disgust-eliciting cues, such features are still more salient in red meat than in other animal products, hence the sequence of meats avoided in the early stages of vegetarianism could reflect the relative availability of disgust stimuli.

The demography of vegetarianism provides additional evidence in support of the possibility that the practice begins with a disgust response to meat. Among Western vegetarians, women greatly outnumber men (Beardsworth \& Bryman, 1999; Neumark-Sztainer, Story, Resnick, \& Blum, 1997; Worsley \& Skrzypiec, 1998), and even nonvegetarian Western women eat considerably less meat than men as a proportion of the diet (Beardsworth \& Bryman, 1999; Fraser, Welch, Luben, Bingham, \& Day, 2000; Perl, Mandic, Primorac, Klapec, \& Perl, 1998; Richardson, Shepherd, \& Elliman, 1993). Correspondingly, in the US, Japan, Indonesia, and the Netherlands women and girls exhibit lower thresholds for the elicitation of disgust (Druschel \& Sherman, 1999; Haidt et al., 1994; Koukounas \& McCabe, 1997; Oppliger \& Zillmann, 1997; Quigley, Sherman, \& Sherman, 1997; first author's field notes; J. Haidt personal communication). ${ }^{3}$ In a sample of New York college students, Mooney and Walbourn (2001) found that, among women, those who avoid meat express significantly greater disgust toward it than those who do not avoid meat, but no such difference exists between male meat-avoiders and male meat eaters. Kubberod et al. (2002) found that Norwegian women are in general more likely to express disgust toward meat than are men, a pattern that is duplicated in the ethnographic literature: Simoons (1994, 323) summarizes two cases of acculturation, the Siberian Yukaghir and the Hawaiian Japanese, in which men adopted novel meat foods while women resisted, expressing revulsion (but see also Pliner \& Pelchat, 1991). Similarly, Aunger (2000) explains the large number of idiosyncratic 'personal [meat] taboos' possessed by women in the Ituri

\footnotetext{
${ }^{2}$ Unpublished data indicate that the road to vegetarianism sometimes begins with avoidance of meat from young animals, behavior which does not fit easily into the disgust-as-motive pattern (P. Rozin, personal communication).

${ }^{3}$ For a discussion of the possible biological factors underlying the sex difference in disgust as it relates to meat eating, see Fessler (2001).
} 
forest as a product of females' greater propensity to experience disgust.

\section{A test of the relationship between meat eating and disgust sensitivity}

Although the evidence described above is suggestive, it is all either anecdotal or indirect. We therefore sought a more conclusive means of determining whether people become disgusted by meat because they are moral vegetarians, or become moral vegetarians because they are disgusted by meat. One approach would be to longitudinally track meat consumption, disgust responses to meat, and beliefs about meat eating in order to establish the sequence with which each of these three factors changes in those individuals who eventually become moral vegetarians. However, moral vegetarianism is of interest as a test case for theories of moral reasoning precisely because it is a minority practice in Western societies. In addition, moral vegetarianism may be adopted over a prolonged period. A longitudinal study would, therefore, necessitate following a large sample of individuals for a long time, a costly enterprise. We therefore adopted an alternative strategy.

The emotivist explanation of moral vegetarianism is premised on the presence of interindividual differences in the propensity to experience spontaneous disgust reactions to meat. As evident in the descriptive material discussed earlier, disgust reactions to meat seem to involve a number of different semantic domains in which disgust can be experienced, including contact with animals, contamination of food, exposure to death, and witnessing violations of the body envelope. Psychological research indicates that, within such domains, disgust reactivity (i.e. ease of elicitation and intensity of response) is highly correlated across types of stimuli: for example, people who are strongly disgusted by seeing an open abdominal wound are also strongly disgusted by seeing someone stick a fish hook through their finger (Haidt et al., 1994). The emotivist explanation of moral vegetarianism therefore generates the prediction that, compared to both health vegetarians and non-vegetarians, moral vegetarians should respond with greater disgust to stimuli involving food, death, and so on. Research also indicates that the propensity to experience disgust is somewhat correlated across semantic domains, making it reasonable to conceptualize general disgust sensitivity as a relatively unified personality trait (Druschel \& Sherman, 1999; Haidt et al., 1994; Quigley et al., 1997; Rozin et al., 1999b). The emotivist hypothesis therefore also generates the prediction that moral vegetarians will exhibit greater overall disgust sensitivity than health vegetarians or nonvegetarians.

While the above predictions constitute a first step toward a test of the emotivist hypothesis, alone they are insufficient. At present, it is not known to what extent disgust sensitivity is stable over time, nor is it known how changes in disgust sensitivity in one domain affect disgust sensitivity in other domains. ${ }^{4}$ It is conceivable that if, as per Rozin et al.'s account, moral vegetarians acquire strong feelings of disgust toward meat following the adoption of moral vegetarian tenets, this change could cause an increase either in the intensity with which disgust is elicited by non-meat stimuli in the relevant domains (food, death, etc.), or in overall disgust sensitivity, or both (P. Rozin, personal communication). Should this occur, simply comparing moral vegetarians, health vegetarians, and non-vegetarians with regard to disgust sensitivity or its domain-specific components might fail to distinguish between the two competing hypotheses, since both accounts, for example, could explain a pattern in which moral vegetarians were found to be more easily or more intensely disgusted than others.

It is possible to circumvent the problem that the stability of disgust sensitivity is as yet unknown by also examining the relationship between disgust sensitivity and the amount of meat consumed on a regular basis. The emotivist hypothesis predicts that disgust sensitivity and meat consumption should constitute inverse continua: individuals exhibiting low disgust sensitivity are predicted to consume lots of meat (and lack negative beliefs about meat eating), those exhibiting an intermediate level of disgust sensitivity are predicted to consume a modest amount of meat (and hold a variety of beliefs), and those exhibiting high disgust sensitivity are predicted to avoid meat (and hold negative beliefs about meat eating). In contrast, the traditional view of the relationship between moral reasoning and emotion does not predict any sort of correlation between disgust sensitivity and meat consumption among individuals who are not moral vegetarians. Accordingly, examining the relationship between these factors in a sample composed of individuals who span the spectrum of meat consumption can distinguish between the competing hypotheses. In order to test these predictions, we therefore designed a survey that independently gauges meat consumption and disgust sensitivity, and also collects information on reasons for meat avoidance.

Because we were interested in assessing behavior across the full spectrum of meat consumption, we sought a sample which was both large and characterized by a greater diversity of ages and backgrounds than is typical of university students. Research conducted via the World Wide Web has the advantage of reaching a large number of potential participants and capturing significant diversity with regard to age and background (Davis, 1999; Krantz \& Dalal, 2000; Reips, 2000). Although the conditions under which web-based measures are administered are less

\footnotetext{
${ }^{4}$ A sample of 34 students tested over a period of 2-4 months exhibited considerable short-term stability in disgust sensitivity, with a test-retest correlation of 0.79 (Rozin et al., 1999b). Stability is likely somewhat less over longer periods since, as will be discussed below, disgust sensitivity declines with age.
} 
controlled than those typical of paper-and-pencil methods, studies employing the same instruments on the web and in person indicate that properly designed web-based versions perform well (Buchanan \& Smith, 1999; Davis, 1999). Overall, well formulated web-based instruments compare favorably with in person methods (Krantz \& Dalal, 2000), with the former possibly having the additional advantage that participants appear to be more forthcoming in computer-mediated surveys, possibly due to greater perceived anonymity (Davis, 1999; Locke \& Gilbert, 1995). For these reasons, we designed our study to be conducted via the web.

\section{Methods}

We created a web-based survey consisting of two parts. A questionnaire (see Appendix A) asked participants to report the frequency with which they had eaten each of a wide variety of meats in the preceding week. In addition to data on age and gender, information on religious affiliation was also collected in order to allow us to control the influence of conventional food proscriptions. At the end of the questionnaire, participants who had indicated that they did not eat three or more of the meats listed were asked to select one or more from the following list of reasons: taste, smell, appearance, ethical reasons, environmental reasons, and health reasons. These same individuals were also asked whether (and, if so, how often) they try to persuade others not to eat meat. Clicking on a button labeled NEXT PAGE at the bottom of this web page then led the participant to a web version of the Disgust Scale (D-Scale) (Haidt et al., 1994). The D-Scale is a widely used measure of disgust sensitivity composed of eight subscales (food, animals, body products, sex, body envelope violations, death, hygiene, and magical thinking), items on which primarily relate to visceral and sensory reactions (off-putting taste combinations, unclean food, frightening vermin, witnessing an injurious accident, etc.) rather than moral or intellectual judgments.

Because moral vegetarianism is associated with complex ideologies, many of which explicitly involve disgust, we took a number of steps to disguise the goals of the investigation so as to reduce the extent to which the instrument elicited well-learned schemas, or prompted attempts to maintain the impression of consistent adherence to an ideological platform. First, nowhere were vegetarianism or related practices (e.g. veganism) explicitly referred to. ${ }^{5}$ Second, the meat consumption index was designed so that it resembled common health surveys, appearing to measure weekly dietary fat consumption rather than meat eating per se. Third, the meat consumption

\footnotetext{
5 This tactic has the additional benefit of avoiding problems caused by the fact that the actual extent of meat avoidance varies within the class of individuals who identify themselves as vegetarians (Janda \& Trocchia, 2001).
}

section and the disgust sensitivity section of the instrument, though linked, were presented as separate surveys-the surveys appeared on separate web pages, used different graphic layouts, and each asked for the participant's age and gender. Fourth, these two surveys were bundled with a third survey (on motion sickness susceptibility, a topic vaguely related to both food consumption and disgust), again using a separate web page and different graphics, and again requesting age and gender. Fifth, both promotional materials (see below) and the 'Information Sheet' web page that preceeded the instrument described the site as containing multiple surveys rather than consisting of a single instrument.

Participants were recruited through a link labeled 'Surveys on diet, disgust, and motion sickness' posted on the American Psychological Society's Psychological Research on the Net web site, and through written requests for volunteer participants sent to listservs such as Psych-L, Ethology, and Anthro-L, as well as The Human Nature Daily Review. These notices used the same study title as the posted link, and provided no additional information about the content or goals of the study. Participation was wholly anonymous. No compensation was offered.

\section{Results}

One thousand three hundred and forty individuals responded to our requests for volunteer participants. We received 53 unsolicited emails from persons interested in the goals of the studies; we responded by stating that results and discussion would be posted on the first author's web site upon completion. None of these correspondents correctly inferred our objective.

In order to screen out frivolous responses, we set a high standard for inclusion in the sample. Data were discarded if (a) age and gender did not match across all three surveys; (b) any part of any of the surveys was left blank (a considerable hurdle given the combined length of the three surveys); and (c) any part of any of the surveys was filled out in an obviously haphazard manner (i.e. claiming both intense susceptibility to motion sickness and complete immunity from seasickness, etc.). After discarding data from 395 individuals for one or more of these reasons, we were left with a sample of 945 participants (326 men and 619 women), with an age range of $13-79$ and a mean age of 30 .

With regard to meat consumption, we found that there was a small but significant negative correlation with age $(r=-0.082, p=0.016)$. Women in our sample consumed meat significantly less often than men $(M=10.8 \pm 0.4, F=9.8 \pm 0.3, t=2.04, p=0.042)$, an effect driven by lesser red meat consumption $(M=3.4 \pm 0.2, F=2.8 \pm 0.1, t=3.03, p=0.002)^{6}$

\footnotetext{
${ }^{6}$ In keeping with recent advertising and public service campaigns, we define red meat as beef, lamb, or sausage, i.e. flesh foods other than poultry, fish or pork.
} 
Table 1

Relationships between reasons for avoiding meat

\begin{tabular}{|c|c|c|c|c|c|c|}
\hline & $\begin{array}{l}\text { Appearance } \\
(n=100, \\
12.8 \% \text { of sample })\end{array}$ & $\begin{array}{l}\text { Environmental } \\
(n=144, \\
18.5 \% \text { of sample })\end{array}$ & $\begin{array}{l}\text { Ethical } \\
(n=99 \\
12.7 \% \text { of sample })\end{array}$ & $\begin{array}{l}\text { Health } \\
(n=395, \\
50.7 \% \text { of sample })\end{array}$ & $\begin{array}{l}\text { Smell } \\
(n=89, \\
11.4 \% \text { of sample })\end{array}$ & $\begin{array}{l}\text { Taste } \\
(n=347, \\
44.5 \% \text { of sample })\end{array}$ \\
\hline Appearance & $26.0^{\mathrm{a}}$ & 6.3 & 23.2 & 9.4 & 13.5 & 17.3 \\
\hline Environmental & 9.0 & $28.5^{\mathrm{a}}$ & 61.6 & 20.5 & 44.9 & 6.1 \\
\hline Ethical & 23.0 & 42.4 & $25.3^{\mathrm{a}}$ & 18.5 & 19.1 & 8.6 \\
\hline Health & 37.0 & 56.3 & 73.7 & $56.5^{\mathrm{a}}$ & 34.8 & 27.1 \\
\hline Smell & 40.0 & 3.5 & 17.2 & 7.8 & $13.5^{\mathrm{a}}$ & 21.0 \\
\hline Taste & 60.0 & 14.6 & 30.3 & 23.8 & 82.0 & $54.5^{\mathrm{a}}$ \\
\hline
\end{tabular}

$N=779$. Columns classify individuals on the basis of reasons for meat avoidance. Rows indicate the percentage of individuals who listed the reason in the column that also listed the reason in the row.

a The percentage of individuals listing the column reason that listed it as their sole reason for meat avoidance.

We found two clear trends with regard to disgust sensitivity, namely that women were more disgust sensitive than men $(M=12.8 \pm 0.3, F=16.3 \pm 0.2, t=11.26$, $p<0.000)$, and disgust sensitivity declined with age $(r=-0.275, p<0.000)$.

Turning to the last questions on the meat survey, 779 individuals listed one or more reasons for not having eaten within the preceding week three or more of the meat items listed. As illustrated in Table 1, many of the individuals who indicated ethical reasons also selected environmental reasons, and a similar clustering occurred with regard to appearance, smell, and taste. There was also substantial association between both ethical and environmental reasons and health, and a lesser clustering of health with taste.

In order to clearly test the hypotheses at issue, we sought to identify three wholly distinct categories of meat-avoiders, namely those motivated exclusively by health concerns $(N=223)$, those motivated exclusively by ethical and/or environmental concerns $(N=80)$, and those who avoided meat items solely because they disliked the taste $(N=189)$. A one way analysis of variance (ANOVA) revealed an age difference between these three categories $\left(F_{(2487)}=17.9\right.$, $p<0.0001)$. A Bonferroni post hoc test showed that this effect was driven by a significant difference between the ages of those who listed health $(32.8 \pm 0.9)$ and those who listed taste $(26.5 \pm 0.7 ; p<0.0001)$ and those who listed ethical/environmental concerns $(27.4 \pm 0.9 ; p<0.0003)$.

While analysis revealed that the three groups do not differ significantly with regard to either the frequency of their attempts to persuade others not to eat meat or in how they prefer their red meat prepared (data not shown), each of the three groups is quite distinct with regard to patterns of meat consumption. A one way ANOVA using reasons for meat avoidance as the factor, meat consumption (total, red, or white) as dependent variables, and controlling for the effects of age revealed a significant effect of type of meatavoider on both total meat $\left(F_{(2457)}=19.0, p<0.0001\right)$, red meat $\left(F_{(2457)}=33.7, \quad p<0.0001\right)$, and white meat $\left(F_{(2457)}=7.9, p=0.0004\right)$ consumption (see Table 2 for details). Bonferroni post hoc tests indicated that taste meat-avoiders eat the most meat of the three groups, consuming more white meat $(p=0.0002)$ and more red meat $(p<0.0001)$ than ethical/environmental meat-avoiders, and, consistent with the health hazards of red meat consumption, more red meat $(p=0.0002)$ but not more white meat ( $p=0.495)$ than health meat-avoiders. In turn, health meat-avoiders do not differ significantly in their consumption of red meat from ethical/environmental meatavoiders but, consistent with the common assumption that white meat is less unhealthful than red meat, do eat more of this $(p=0.0008)$ than ethical/environmental meat-avoiders. Self-reported patterns of meat consumption are thus consistent with self-reported reasons for meat avoidance, with taste meat-avoiders singling out particular items which they dislike but otherwise eating a lot of meat, health meatavoiders abstaining from unhealthy red meat but eating white meat, and ethical/environmental meat-avoiders eating little of either type of meat.

Turning to the key question motivating the investigation, across the sample as a whole, controlling for age we found a small positive correlation between total meat consumption and disgust sensitivity $(r=0.103, p=0.003)$. This was due to an increase in white meat consumption with increasing disgust sensitivity $(r=0.129, p<0.000)$. Although red meat consumption was not correlated with overall disgust sensitivity ( $r=0.055, p=0.108$ ), it was

Table 2

Meat consumption (servings per week, Mean $\pm \mathrm{SE}$ ) by reasons for avoiding meat

\begin{tabular}{|c|c|c|c|}
\hline \multirow[t]{2}{*}{ Reason } & \multicolumn{3}{|c|}{ Meat consumption } \\
\hline & Red & White & Total \\
\hline Ethical/environmental & $1.7 \pm 03^{\mathrm{a}}$ & $2.8 \pm 0.5^{\mathrm{b}, \mathrm{a}}$ & $6.6 \pm 0.9^{\mathrm{b}, \mathrm{a}}$ \\
\hline Health & $2.3 \pm 0.1^{\mathrm{a}}$ & $4.4 \pm 0.2^{\mathrm{c}}$ & $9.9 \pm 0.4^{\mathrm{c}, \mathrm{a}}$ \\
\hline Taste & $4.1 \pm 0.2^{\mathrm{b}, \mathrm{c}}$ & $4.7 \pm 0.3^{\mathrm{c}}$ & $11.8 \pm 0.5^{\mathrm{b}, \mathrm{c}}$ \\
\hline
\end{tabular}

\footnotetext{
${ }^{\text {a }}$ Significantly different from taste meat-avoiders $(p<0.0009)$.

b Significantly different from health meat-avoiders $(p<0.003)$.

${ }^{c}$ Significantly different from ethical meat-avoiders $(p<0.0009)$.
} 
Table 3

Correlations (Pearson's r) between the consumption of white or red meat and disgust sensitivity, by subscale domain

\begin{tabular}{|c|c|c|c|c|c|c|c|c|c|}
\hline \multirow[t]{2}{*}{ Meat } & \multicolumn{9}{|c|}{ Disgust domain } \\
\hline & Animal & Body products & Death & Envelope violations & Food & Hygiene & Magic & Sex & Total score \\
\hline White & 0.036 & 0.012 & $0.102^{*}$ & 0.041 & $0.084^{*}$ & $0.081^{*}$ & $0.077^{*}$ & $0.170^{* *}$ & $0.129^{* *}$ \\
\hline Red & -0.040 & -0.038 & 0.033 & 0.062 & $0.093^{*}$ & $0.077^{*}$ & 0.027 & $0.163^{* *}$ & 0.055 \\
\hline
\end{tabular}

$* p<0.05, * * p<0.001$.

positively correlated with scores on three of the eight subscales of the D-Scale; white meat consumption was positively correlated with five of the subscales. In both cases, positive correlations included the presumably highly relevant domain of food (see Table 3). Comparing the lowest quartile on the meat consumption index, a group which presumably includes all of the vegetarians in our sample, with the highest quartile ('the carnivores'), the latter are more disgust sensitive than the former $(t=3.402$, $p=0.001)$, a pattern that remains relatively unchanged when meat consumption is compared with scores on the food subscale on the D-Scale (lowest vs highest quartile, $t=3.40, p=0.001$ ).

Comparing ethical/environmental meat-avoiders, health meat-avoiders, and taste meat-avoiders with regard to their respective scores on the D-Scale, controlling for the possible confounding effects of age, no significant differences were found in overall disgust sensitivity (see Table 4). Comparing the three classes of meat-avoiders with regard to their respective subscale scores, again controlling for age, a one way ANOVA showed a significant effect only for the sex domain $\left(F_{(2457)}=3.1, p=0.045\right)$ (see Table 4). Bonferroni's post hoc test revealed that the effect was driven by the disparity on this subscale between taste meat-avoiders and health meat-avoiders $(p=0.0005)$.

Finally, Spearman's rank correlation test revealed a positive relationship between disgust sensitivity and preferred type of red meat preparation (ranging from rare [least cooked] to well done [most cooked] $)(r=0.244$, $p<0.0001)$. Further analyses indicated that this effect was driven by a significantly positive relationship between degree of cooking and all disgust subscales except the food domain, with the death subscale showing the strongest correlation (see Table 5).

\section{Discussion}

Our findings regarding general demographic patterns parallel those of other investigators. Meat consumption is negatively correlated with age (cf. Aranceta et al., 1998; Hudy, Caster, \& Hames, 1985; Von Post-Skagegard et al., 2002), and women eat meat less frequently than men, (Beardsworth \& Bryman, 1999; Fraser et al., 2000; Perl et al., 1998; Richardson et al., 1993), a difference which is primarily due to lower rates of red meat consumption (cf. Baghurst, 1999; Smit, Nieto, Crespo, \& Mitchell, 1999). Likewise, our findings that (a) women are more disgust sensitive than men, and (b) disgust sensitivity decreases with age replicate previous studies which used smaller samples and in person administration of the D-Scale (Haidt et al., 1994; Koukounas \& McCabe, 1997; Oppliger \& Zillmann, 1997; Quigley et al., 1997).

Exploring reasons for meat avoidance, the close association which we found between ethical and environmental reasons is consistent with the overlap among factors such as animal cruelty, ecological conservation, and humanitarianism present in many of the prevailing ideological rationales for vegetarianism. Likewise, the linkages between appearance, smell, and taste that appear in our data

Table 4

Disgust domain subscale scores (Mean \pm SE) by reasons for avoiding meat

\begin{tabular}{|c|c|c|c|c|c|c|c|c|c|}
\hline \multirow[t]{2}{*}{ Reason } & \multicolumn{9}{|c|}{ Disgust domain } \\
\hline & Animal & Body products & Death & Envelope violations & Food & Hygiene & Magic & Sex & Total score \\
\hline Ethical & $2.05 \pm 0.12$ & $2.46 \pm 0.11$ & $1.34 \pm 0.13$ & $2.01 \pm 0.10$ & $1.86 \pm 0.09$ & $1.30 \pm 0.10$ & $1.43 \pm 0.11$ & $2.23 \pm 0.11^{\mathrm{a}}$ & $14.7 \pm 0.5$ \\
\hline Health & $2.27 \pm 0.07$ & $2.37 \pm 0.07$ & $1.18 \pm 0.08$ & $1.91 \pm 0.05$ & $1.87 \pm 0.05$ & $1.56 \pm 0.07$ & $1.47 \pm 0.07$ & $2.20 \pm 0.06^{\mathrm{c}}$ & $14.9 \pm 0.3$ \\
\hline Taste & $2.34 \pm 0.08$ & $2.47 \pm 0.07$ & $1.42 \pm 0.09$ & $1.97 \pm 0.06$ & $1.85 \pm 0.06$ & $1.59 \pm 0.07$ & $1.59 \pm 0.07$ & $2.54 \pm 0.07^{\mathrm{b}, \mathrm{c}}$ & $15.9 \pm 0.3$ \\
\hline
\end{tabular}

a Significantly different from taste meat-avoiders $(p<0.05)$.

b Significantly different from health meat-avoiders $(p<0.05)$.

c Significantly different from ethical meat-avoiders $(p<0.05)$. 
Table 5

Spearman rank correlations for meat preparation (rare = minumum degree of cooking, well done $=$ maximum degree of cooking) with disgust subscales

\begin{tabular}{llllllllll}
\hline & \multicolumn{2}{l}{ Disgust domain } & & & & \\
\cline { 2 - 9 } & Animal & Body products & Death & Envelope violations & Food & Hygiene & Magic & Sex & Total score \\
\hline Meat preparation & $0.112^{*}$ & $0.092^{*}$ & $0.212^{* *}$ & $0.160^{* *}$ & 0.008 & $0.199^{* *}$ & $0.164^{* *}$ & $0.197^{* *}$ & $0.244^{* *}$ \\
\hline
\end{tabular}

$* p<0.05, * * p<0.0001$

are congruent with the overlapping contributions of different sensory modalities to food preferences (Kubberod et al., 2002; Tuorila et al., 1994). The presence of individuals who selected two or more of the three major categories of reasons (ethical/environmental, health, and taste) indicates that, for some participants, meat avoidance is markedly overdetermined (cf. Cooper, Wise, \& Mann, 1985). Lastly, looking exclusively at those individuals who selected only one of the three major reasons for meat avoidance, the significant disparity in age between health meat-avoiders and the other two classes is consistent with published accounts of increases in food-relevant health concerns with age (Reime, Novak, Born, Hagel, \& Wanek, 2000).

Patterns of meat consumption were wholly congruent with self-reported reasons for meat avoidance, with ethical/environmental meat-avoiders eating the least meat, health meat-avoiders eating little red meat but substantial amounts of white meat, and taste meat-avoiders excluding only select disliked meat items from their diet. This clear tripartite division indicates that our method succeeded in identifying distinctly different classes of meat-avoiders. With this as background, we were then able to address the hypotheses at issue.

Contrary to predictions derived from the emotivist hypothesis, across our sample as a whole, meat consumption was positively correlated with overall disgust sensitivity. With regard to subscales of the disgust sensitivity instrument, consumption of both white and red meat was positively correlated with the presumably highly relevant domains of food and hygiene. Likewise, those individuals consuming the most meat were more disgust sensitive, both overall and in the food domain, than those individuals consuming the least meat.

Contrary to predictions derived from the emotivist hypothesis, examining those participants who avoid meat solely on the grounds of either ethical/environmental concerns, health concerns, or taste, we found that these three classes of meat-avoiders did not differ from one another on overall disgust sensitivity. With regard to the subscales of the disgust sensitivity instrument, the only difference found (a) did not differentiate ethical/environmental meat-avoiders from health meat-avoiders, and (b) involved a subscale (sex) of no clear relevance to the question of dietary practice or preference.
The only area in which disgust sensitivity clearly shapes dietary preferences in the expected direction involves red meat preparation, as, consistent with the salience of blood as a disgust stimulus, there is a positive correlation between disgust sensitivity and the thoroughness with which individuals who consume red meat prefer their meat to be cooked.

\section{Limitations}

The many parallels between the results of each of the two parts of our instrument and others' published findings (e.g. the influence of age and gender on both disgust sensitivity and meat consumption, etc.) bolster our confidence in our methods. Nevertheless, this study is subject to a number of potential limitations.

The menu from which participants selected reasons for not having eaten three or more of the meat items listed included neither financial constraints nor dieting behavior, factors that may affect meat consumption (Gilbody, Kirk, \& Hill, 1999; Jeremiah, 1982; Mooney \& Walbourn, 2001). It is reasonable to assume that individuals who avoid meat solely for economic reasons either (a) would not have selected from the list of reasons for meat avoidance, and hence would not have been included in the subsample of 779 individuals, or (b) would have chosen randomly from among the reasons listed, and hence would not have biased the results. It seems likely that individuals who avoided meat for purposes of weight control would be prone to select 'health' from the menu of reasons given, as this is the closest match to dieting. Because individuals prone to dieting may exhibit elevated disgust sensitivity (Harvey et al., 2002), this pattern of responses would have inflated the mean disgust sensitivity among health meat-avoiders. While this could conceivably have interfered with one of the substantive comparisons on which we relied (that between moral meat-avoiders and health meat-avoiders), it would not have affected another key comparison, namely that between moral meat-avoiders and taste meat-avoiders. In addition, given that dieters would not have been present in the highest quartile of meat consumers, the fact that the latter are significantly more disgust sensitive than the lowest quartile of meat consumers indicates that whatever inflating influences dieters may have had on the mean disgust sensitivity of meat-avoiders as a class failed to swamp 
the overarching pattern, namely that meat eating is positively correlated with disgust sensitivity.

A number of other limitations should also be considered in evaluating our study. First, meat consumption varies dramatically along cultural lines. While most of the Internet venues used to publicize our survey are directed primarily at a US audience, some have a more international scope. We have no way of determining the nationality or geographical location of our participants, and even an exclusively North American sample would likely contain considerable cultural heterogeneity. Because we did not collect information on participants' cultural backgrounds, we cannot rule out the possibility that cultural factors confounded our comparison of meat consumption and disgust sensitivity. However, to our knowledge, no existing account of cultural factors predicts the results that we obtained. Second, our meat consumption survey measured frequency rather than absolute amount, and it is possible (though unlikely) that disgust sensitivity shapes the latter more than the former. Third, with regard to both diet and disgust reactivity, surveys may not wholly capture people's actual behavior and experience (Muhlheim, 1996; Rozin et al., 1999b; Smith, Jobe, \& Mingay, 1991). Lastly, the validity of surveys may be reduced by web-based administration, as it is possible that the enhanced ease of participation and perceived greater anonymity may increase frivolous responses (Nosek, Banaji, \& Greenwald, 2002). It is worth noting, however, that (a) our design included measures such as repeat questions intended to aid in identifying frivolous responses, (b) we employed broad criteria in identifying frivolous responses, resulting in a high discard rate, and (c) in identifying targets for discard, with the exception of the age and gender questions, the three surveys were examined in isolation, precluding biased patterns of discarding.

\section{Conclusion}

Previously, Rozin et al. (1997) reported that, compared to those who follow a vegetarian diet for health reasons, individuals who explain their vegetarianism in moral terms express more disgust at meat. Consistent with traditional views of the relationship between emotion and moral reasoning, Rozin et al. argued that moral vegetarians adopt their position on reasoned grounds, and only later become disgusted by meat as a consequence of their moral stance. Recently, investigators have questioned the direction of causality in the relationship between emotion and moral reasoning, arguing that emotions often drive decision-making, with moral rationales being seen as little more than post hoc justifications. To determine which of these approaches best characterizes moral vegetarianism, we conducted a web-based survey of over 900 adults, examining the relationship between a personality trait (disgust sensitivity), self-reported behavior (recent meat consumption), and stated motives (reasons for meat avoidance).

Our results reveal a positive correlation between overall disgust sensitivity and the frequency of meat consumption. This pattern is driven in part by positive correlations between meat consumption and disgust sensitivity in the crucial domains of food and hygiene, and, with regard to white meat consumption, in the death domain. Classifying individuals who did not consume three or more of the meat items listed with regard to their stated reasons for meat avoidance, we found that those who avoid meat for ethical and environmental reasons are no more disgust sensitive than those who avoid meat for health or taste reasons. Hence, in contrast to suggestive ethnographic and demographic evidence, our findings challenge an emotivist account in which adherence to moral vegetarianism is seen as stemming from greater disgust reactions to meat. We therefore conclude that Rozin et al. are correct in their interpretation of their finding that moral vegetarians evince greater disgust at meat: at least in this case, disgust is a consequence of, rather than causal of, the adoption of a moral position.

\section{Acknowledgements}

We thank John Krantz (APS) and Ian Pitchford (HumanNature.com) for assistance in publicizing the online studies, Paul Rozin and an anonymous reviewer for helpful feedback on the manuscript, and Jonathan Haidt for useful suggestions. This research was supported by a Director's Grant, Neuropsychiatric Institute, UCLA.

\section{Appendix A. A facsimile of the on-line food frequency questionnaire}

\begin{tabular}{ll}
\hline Servings \\
per week
\end{tabular}

Dairy products

Non-fat milk

Lowfat milk

Whole milk

Meats and high

protein products

Fish and shellfish

Sushi (raw fish)

Chicken without skin

Chicken with skin 


\section{Servings}

per week

Turkey without skin

Turkey with skin

Lean beef

Higher fat beef

Lean pork

Higher fat pork

Higher fat veal

Higher fat lamb

Cold cuts

Hot dogs

Sausages

Tofu and soy products

Beans

Scrambled eggs

Sunny side up eggs

Hard-boiled eggs

Nuts

General information

Age

Sex

Religion
Please select

from list

Rare

Medium rare

Medium

Medium well

Well done

Please select

from list

Rare

Medium rare

Medium

Medium well

Well done
Appearance

Ethical reasons

Health reasons

Environmental reasons

2) Do you try to persuade other people not to eat meat?

Never

Occasionally

Often

Whenever I can

\section{NEXT PAGE}

\section{References}

Adams, C. J. (2000). The sexual politics of meat: A feminist-vegetarian critical theory (10th anniversary ed.). New York: Continuum.

American Dietetic Association (A.D.A.) (1997). Position of the American Dietetic Association: vegetarian diets. Journal of the American Dietetic Association, 97, 1317-1321.

Angyal, A. (1941). Disgust and related aversions. Journal of Abnormal \& Social Psychology, 36, 393-412.

Aranceta, J., Perez Rodrigo, C., Eguileor, I., Marzana, I., Gonzalez de Galdeano, L., \& Saenz de Buruaga, J. (1998). Food consumption patterns in the adult population of the Basque Country (EINUT-I). Public Health Nutrition, 1, 185-192.

Aunger, R. (2000). The life history of culture learning in a face-to-face society. Emos, 28, 1-38.

Back, K. W., \& Glasgow, M. (1981). Social networks and psychological conditions in diet preferences: gourmets and vegetarians. Basic \& Applied Social Psychology, 2, 1-9.

Baghurst, K. (1999). Red meat consumption in Australia: intakes, contributions to nutrient intake and associated dietary patterns. European Journal of Cancer Prevention, 8, 185-191.

Barkow, J. H., Taslilm, N. A., Hadju, V., Ishak, E., Attamimi, F., Silwana, S., Dachlan, D. M., Ramli, \& Yahya, A. (2001). Social competition, social intelligence, and why the Bugis know more about cooking than about nutrition. Proceedings of the British Academy, 110, 119-147.

Beardsworth, A., \& Bryman, A. (1999). Meat consumption and vegetarianism among young adults in the UK. British Food Journal, 101, 289-300.

Beardsworth, A., \& Keil, T. (1992). The vegetarian option: varieties, conversions, motives and careers. Sociological Review, 40, 253-293.

Bourdieu, P. (1984). Distinction: A social critique of the judgement of taste. Cambridge, MA: Harvard University Press.

Buchanan, T., \& Smith, J. L. (1999). Using the Internet for psychological research: personality testing on the World Wide Web. British Journal of Psychology, 90, 125-144.

Chagnon, N. A. (1997). Yanomamö (5th ed.). Fort Worth: Harcourt Brace College Publishers.

Cooper, C., Wise, T. N., \& Mann, L. S. (1985). Psychological and cognitive characteristics of vegetarians. Psychosomatics, 26, 521-523. see also pages 526, 527.

Cosmides, L., \& Tooby, J. (2000). Evolutionary psychology and the emotions. In M. Lewis, \& J. M. Haviland-Jones (Eds.), Handbook of emotions (2nd ed.) (pp. 91-115). New York: Guilford Press.

Curtis, V., \& Biran, A. (2001). Dirt, disgust, and disease: is hygiene in our genes? Perspectives in Biology and Medicine, 44, 17-31.

Damasio, A. R. (1994). Descartes' error: Emotion, reason, and the human brain. New York: Putnam.

Davis, R. N. (1999). Web-based administration of a personality questionnaire: comparison with traditional methods. Behavior Research Methods, Instruments \& Computers, 31, 572-577. 
Druschel, B. A., \& Sherman, M. F. (1999). Disgust sensitivity as a function of the Big Five and gender. Personality \& Individual Differences, 26, 739-748.

Dwyer, J. T., Mayer, L. D. V. H., Dowd, K., Kandel, R. F., \& Mayer, J. (1974). The new vegetarians: the natural high? Journal of the American Dietetic Association, 65, 529-536.

Fallon, A. E., \& Rozin, P. (1983). The psychological bases of food rejections by humans. Ecology of Food and Nutrition, 13, 15-26.

Fessler, D. M. T. (2001). Luteal phase immunosuppression and meat eating. Rivista di Biologia/Biology Forum, 94, 407-430.

Fessler, D. M. T., \& Navarrete, C. D. (2003). Meat is good to taboo: dietary proscriptions as a product of the interaction of psychological mechanisms and social processes. Journal of Cognition \& Culture, 3, $1-40$

Fiddes, N. (1991). Meat, a natural symbol. New York: Routledge.

Fraser, G. E., Welch, A., Luben, R., Bingham, S. A., \& Day, N. E. (2000) The effect of age, sex, and education on food consumption of a middleaged English cohort-EPIC in East Anglia. Preventive Medicine, 30, 26-34.

Gilbody, S. M., Kirk, S. F. L., \& Hill, A. J. (1999). Vegetarianism in young women: another means of weight control? International Journal of Eating Disorders, 26, 87-90.

Greene, J. D., Sommerville, R. B., Nystrom, L. E., Darley, J. M., \& Cohen, J. D. (2001). An fMRI investigation of emotional engagement in moral judgment. Science (Washington DC), 293, 2105-2108.

Haidt, J. (2001). The emotional dog and its rational tail: a social intuitionist approach to moral judgment. Psychological Review, 108, 814-834.

Haidt, J., Koller, S. H., \& Dias, M. G. (1993). Affect, culture, and morality, or is it wrong to eat your dog? Journal of Personality \& Social Psychology, 65, 613-628.

Haidt, J., McCauley, C., \& Rozin, P. (1994). Individual differences in sensitivity to disgust: a scale sampling seven domains of disgust elicitors. Personality \& Individual Differences, 16, 701-713.

Harvey, T., Troop, N. A., Treasure, J. I. \& Murphy, T. (2002). Fear, disgust, and abnormal eating attitudes: A preliminary study. International Journal of Eating Disorders, 32, 213-218.

Hudy, S. P., Caster, W. O., \& Hames, C. G. (1985). Changes in food and nutrient intake patterns observed in older men and women in Evans County, Georgia, [USA]. Journal of Clinical and Experimental Gerontology, 7, 99-114.

Jabs, J., Devine, C. M., \& Sobal, J. (1998). Model of the process of becoming a vegetarian: health vegetarians and ethical vegetarians. Journal of Nutrition Education, 30, 196-202.

Janda, S., \& Trocchia, P. J. (2001). Vegetarianism: toward a greater understanding. Psychology \& Marketing, 18, 1205-1240.

Jeremiah, L. E. (1982). A review of factors influencing consumption, selection, and acceptability of meat purchases. Journal of consumer studies and home economics, 6, 137-154.

Kenyon, P. M., \& Barker, M. E. (1998). Attitudes towards meat-eating in vegetarian and non-vegetarian teenage girls in England: an ethnographic approach. Appetite, 30, 185-198.

Kohlberg, L. (1984). The psychology of moral development: The nature and validity of moral stages (1st ed.). San Francisco: Harper \& Row.

Koukounas, E., \& McCabe, M. (1997). Sexual and emotional variables influencing sexual response to erotica. Behaviour Research \& Therapy, $35,221-230$.

Krantz, J. H., \& Dalal, R. (2000). Validity of Web-based psychological research. In M. H. Birnbaum (Ed.), Psychological experiments on the Internet (pp. 35-60). San Diego, CA: Academic Press.

Kubberod, E., Ueland, O., Tronstad, A., \& Risvik, E. (2002). Attitudes towards meat and meat-eating among adolescents in Norway: a qualitative study. Appetite, 38, 53-62.

Kurtines, W. M., \& Gewirtz, J. L. (1991). Handbook of moral behavior and development. Hillsdale, NJ: L. Erlbaum.

Lapsley, D. K. (1996). Moral psychology. Boulder, CO: Westview Press.

Levy, R. I. (1973). Tahitians: Mind and experience in the Society Islands. Chicago: University of Chicago Press.
Locke, S. D., \& Gilbert, B. O. (1995). Method of psychological assessment, self-disclosure, and experiential differences: a study of computer, questionnaire, and interview assessment formats. Journal of Social Behavior \& Personality, 10, 255-263.

Mooney, K. M., \& Walbourn, L. (2001). When college students reject food: not just a matter of taste. Appetite, 36, 41-50.

Muhlheim, L. S (1996). Mechanisms of underreporting of dietary intake. Unpublished thesis, Rutgers the State U New Jersey

Neumark-Sztainer, D., Story, M., Resnick, M. D., \& Blum, R. W. (1997). Adolescent vegetarians: a behavioral profile of a school-based population in Minnesota. Archives of Pediatrics \& Adolescent Medicine, 151, 833-838.

Nisbett, R. E., \& Wilson, T. D. (1977). Telling more than we can know: verbal reports on mental processes. Psychological Review, 84, $231-259$.

Nosek, B. A., Banaji, M. R., \& Greenwald, A. G. (2002). E-research: ethics, security, design, and control in psychological research on the Internet. Journal of Social Issues, 58, 161-176.

Oppliger, P. A., \& Zillmann, D. (1997). Disgust in humor: its appeal to adolescents. Humor: International Journal of Humor Research, 10, $421-437$.

Perl, M. A., Mandic, M. L., Primorac, L., Klapec, T., \& Perl, A. (1998). Adolescent acceptance of different foods by obesity status and by sex. Physiology \& Behavior, 65, 241-245.

Pliner, P., \& Pelchat, M. L. (1991). Neophobia in humans and the special status of foods of animal origin. Appetite, 16, 205-218.

Quigley, J. F., Sherman, M. F., \& Sherman, N. C. (1997). Personality disorder symptoms, gender, and age as predictors of adolescent disgust sensitivity. Personality \& Individual Differences, 22, 661-667.

Reime, B., Novak, P., Born, J., Hagel, E., \& Wanek, V. (2000). Eating habits, health status, and concern about health: a study among 1641 employees in the German metal industry. Preventive Medicine: An International Journal Devoted to Practice \& Theory, 30, 295-301.

Reips, U.-D. (2000). The Web experiment method: Advantages, disadvantages, and solutions. In M. H. Birnbaum (Ed.), Psychological experiments on the Internet (pp. 89-117). San Diego, CA: Academic Press.

Richardson, N. J., Shepherd, R., \& Elliman, N. A. (1993). Current attitudes and future influences on meat consumption in the UK. Appetite, 21, 41-51.

Rozin, P. (1999). Food is fundamental, fun, frightening, and far-reaching. Social Research, 66, 9-30.

Rozin, P., Fischler, C., Imada, S., Sarubin, A., \& Wrzesniewski, A. (1999a). Attitudes to food and the role of food in life in the USA, Japan, Flemish Belgium and France: possible implications for the diet-health debate. Appetite, 33, 163-180.

Rozin, P., Haidt, J., \& McCauley, C. R. (1993). Disgust. In M. Lewis, \& J. M. Haviland (Eds.), Handbook of emotions (pp. 575-594). New York: The Guilford Press.

Rozin, P., Haidt, J., \& McCauley, C. R. (1999c). Disgust: The body and soul emotion. In T. Dalgleish, \& M. J. Power (Eds.), Handbook of cognition and emotion (pp. 429-445). Chichester, England: Wiley.

Rozin, P., Haidt, J., McCauley, C., Dunlop, L., \& Ashmore, M. (1999b). Individual differences in disgust sensitivity: comparisons and evaluations of paper-and-pencil versus behavioral measures. Journal of Research in Personality, 33, 330-351.

Rozin, P., Markwith, M., \& Stoess, C. (1997). Moralization and becoming a vegetarian: the transformation of preferences into values and the recruitment of disgust. Psychological Science, 8, 67-73.

Rozin, P., \& Singh, L. (1999). The moralization of cigarete smoking in the United States. Journal of Consumer Psychology, 8, 339-342.

Santos, M. L. S., \& Booth, D. A. (1996). Influences on meat avoidance among British students. Appetite, 27, 197-205.

Simoons, F. J. (1994). Eat not this flesh: Food avoidances from prehistory to the present (2nd ed.). Madison: University of Wisconsin Press.

Smit, E., Nieto, F. J., Crespo, C. J., \& Mitchell, P. (1999). Estimates of animal and plant protein intake in US adults: results from the Third 
National Health and Nutrition Examination Survey, 1988-1991. Journal of the American Dietetic Association, 99, 813-820.

Smith, A. F., Jobe, J. B., \& Mingay, D. J. (1991). Retrieval from memory of dietary information. Applied Cognitive Psychology, 5, 269-296.

Tuorila, H., Meiselman, H. L., Bell, R., Cardello, A. V. \& Johnson, W. (1994). Role of sensory and cognitive information in the enhancement of certainty and liking for novel and familiar foods. Appetite, 23, 231-246.

Turiel, E. (1983). The development of social knowledge: Morality and convention. New York: Cambridge University Press.
Twigg, J. (1979). Food for thought: purity and vegetarianism. Religion, 9 , 13-33.

Von Post-Skagegard, M., Samuelson, G., Karlstrom, B., Mohsen, R., Berglund, L., \& Bratteby, L. E. (2002). Changes in food habits in healthy Swedish adolescents during the transition from adolescence to adulthood. European Journal of Clinical Nutrition, 56, 532-538.

Webster, A. J. F. (1994). Meat and right: the ethical dilemma. Proceedings of the Nutrition Society, 53, 263-270.

Worsley, A., \& Skrzypiec, G. (1998). Teenage vegetarianism: prevalence, social and cognitive contexts. Appetite, 30, 151-170. 